\title{
ONECUT2 many towards AR-independence
}

Androgen receptor (AR) dependence is a defining characteristic of many metastatic castration-resistant prostate cancers (mCRPCs), but some aggressive tumours are ARindependent and rely on alternative transcriptional pathways. A new study has identified one cut domain family member 2 (ONECUT2) as a transcription factor that negatively regulates $\mathrm{AR}$ networks and drives AR-independent transcriptional programmes in mCRPC.

The investigators - led by Mirja Rotinen, Sungyong You and Michael Freeman — initially used a bioinformatics model of transcription factor activity and network relationships integrating transcriptomics data from a prostate cancer discovery cohort to identify ONECUT2 as a prominent transcriptional node that is highly active in mCRPC. "From there, we used genetic approaches in model systems, additional bioinformatics studies in large cohorts and preclinical models to understand the functional significance of ONECUT2," explains Freeman.

Transcriptomic and bioinformatic analyses in mCRPC cell line models

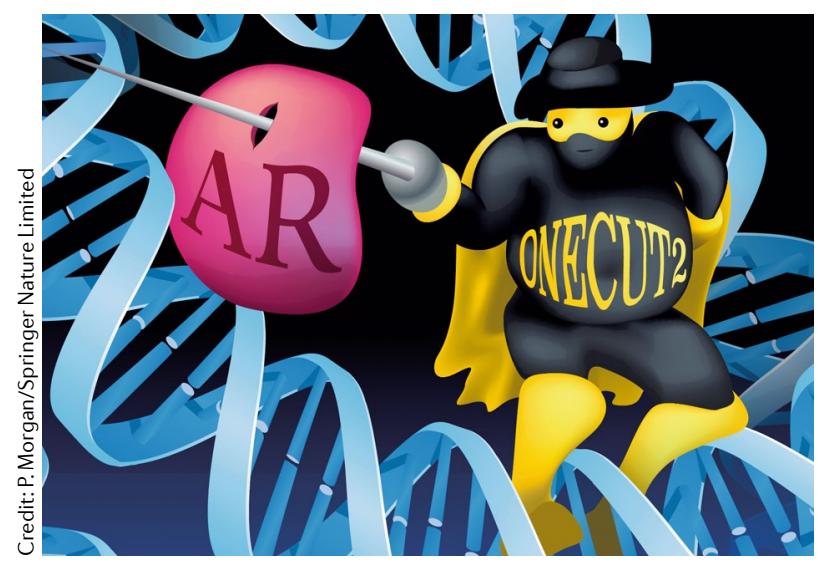

revealed that ONECUT2 repressed $\mathrm{AR}$ and $\mathrm{AR}$ target gene expression. Importantly, ONECUT2 transcriptional activity — inferred using a bioinformatics approach - inversely correlated with an AR transcriptomic signature in the discovery cohort, suggesting that ONECUT2 antagonizes AR and its transcriptional programme. Furthermore, multiplex immunofluorescence microscopy coupled to digital pathology in high-grade prostate cancer tissues revealed that nuclear ONECUT2 and AR expression were inversely correlated in $\mathrm{ONECUT}^{+} \mathrm{AR}^{+}$cells.

Comparison of ONECUT2 chromatin immunoprecipitation and sequencing (ChIP-seq) data from mCRPC cell lines with published AR ChIP-seq data showed that ONECUT2 and AR share 2,151 common regulatory targets. ONECUT2 bound to 48 of 101 tested AR-regulated genes, of which 41 contained shared ONECUT2-AR binding sites in their promoters. Gene set enrichment analysis showed that enforced ONECUT2 expression in mCRPC cells markedly repressed the majority of the ONECUT2-AR target genes, whereas ONECUT2 silencing only moderately de-repressed these genes. These data suggest that ONECUT2 functionally antagonizes and directly suppresses a subset of AR-regulated genes.

Experiments also revealed that ONECUT2 represses expression of forkhead box protein A1 (FOXA1), a frequently lost transcription factor that inhibits neuroendocrine prostate cancer (NEPC) differentiation in mCRPC, suggesting that ONECUT2 mediates NEPC phenotypic conversion. Indeed, ONECUT2 expression positively correlated with an NEPC differentiation signature in MCRPC tissue and was higher in NEPC tumours than in adenocarcinoma tissues. These data, together with additional insights from preclinical models, infer that ONECUT2 drives NEPC differentiation.

Analysis of prostate cancer cohorts revealed that ONECUT2 is a clinically relevant therapeutic target in $\mathrm{mCRPC}$. High ONECUT2 expression was associated with biochemical recurrence in pretreatment localized tumour tissues, and inferred ONECUT2 and AR activation were inversely correlated in biopsy tissue samples from high-grade nonmetastatic and metastatic tumours.

Following functional validation experiments showing that ONECUT2 depletion inhibited tumour growth and metastatic potential, the authors tested the growth suppression and antimetastatic effects of a novel ONECUT2 inhibitor. "We used in silico modelling of the structure of ONECUT2 on DNA and chemical library screening to identify a small molecule that directly binds to ONECUT2 and interferes with its ability to bind DNA," explains Freeman. Encouragingly, the compound phenocopied the effects of ONECUT2 depletion in vitro and reduced tumour volume and metastasis in mouse models.

Overall, the findings demonstrate that ONECUT2 is a survival factor and a master regulator of AR networks that drives AR-dependent adenocarcinoma towards NEPC differentiation in aggressive mCRPC subsets. The study also demonstrates the strong translational potential of ONECUT2-targeted therapeutic agents. "We are working to develop chemical derivatives of our lead compound to improve potency and drug-like features," concludes Freeman.

Conor A. Bradley

ORIGINAL ARTICLE Rotinen, M. et al. ONECUT2 is a targetable master regulator of lethal prostate cancer that suppresses the androgen axis. Nat. Med. 24, 1887-1898 (2018) 